\title{
Monte Carlo Uncertainty Analysis for Photothermal Radiometry Measurements Using a Curve Fit Process
}

\author{
Kyle Horne, Austin Fleming, Heng Ban \\ Department of Mechanical and Aerospace Engineering \\ Utah State University \\ 4130 Old Main Hill \\ Logan, UT 84322 \\ E-mail: heng.ban@usu.edu
}

\begin{abstract}
Photothermal Radiometry (PTR) has become a popular method to measure thermal properties of layered materials. Much research has been done to determine the capabilities of PTR, but with little uncertainty analysis. This study performs a Monte Carlo uncertainty analysis to quantify uncertainty of film diffusivity and effusivity measurements, presents a sensitivity study for each input parameter, compares linear and logarithmic spacing of data points on frequency scans, and investigates the validity of a 1-D heat transfer assumption.
\end{abstract}




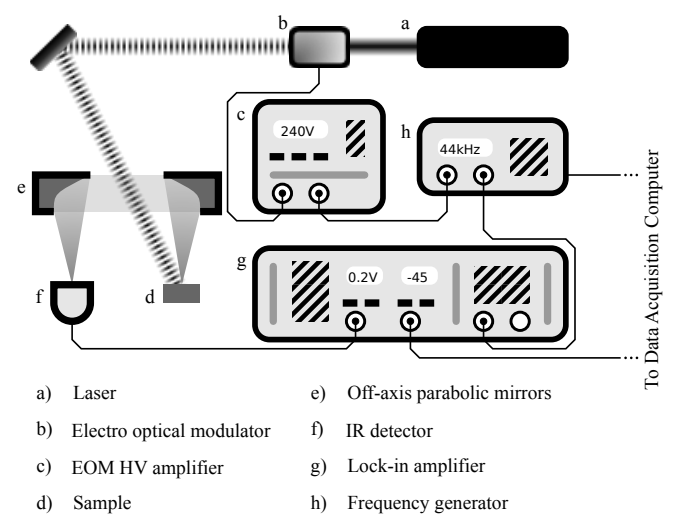

Figure 1: Diagram of PTR system modeled in uncertainty calculations

\section{Introduction}

Originally developed by Nordal and Kanstad[1], Photothermal Radiometry (PTR) is a non-contacting and non-destructive method for determining a sample's thermal properties. PTR uses a light source to induce a thermal response in a sample, which is observed by the infrared radiation emitted from the sample. A theoretical model for the thermal response is then fitted to the data to obtain the thermal properties[2]. Many different thermal excitation and detection methods have been conducted $[3,4,5,6,7]$. However, this study focuses on the frequency-domain front-detection PTR method. This method uses a periodically modulated laser to induce thermal waves in a sample. These thermal waves are reflected and refracted at the layer interfaces, thereby affecting the surface temperature's phase and amplitude. An infrared detector measures radiation emitted from the sample's surface, which is related to the surface temperature by Boltzmann's law. A lock-in amplifier is then used to measure the phase and amplitude of the signal from the infrared detector. A theoretical model is fitted to this amplitude and phase data with thermal diffusivity and effusivity as fit parameters. This method can be applied used to measure layered samples' thermal properties. The diagram in figure 1 shows the theoretical PTR setup being studied in this work. This uncertainty work is based on Photothermal Radiometry, however the results and methods used can also be applied to other frequency domain photothermal methods. Some of these methods include Modulated Optical Reflectance, Photodeflectance, Photo-Pyroelectric, Photoacoustic, and Photothermal Displacement[8, 9, 10, 11, 12].

When conducting an experiment it is important to estimate and report the uncertainty of the results; it is also necessary to report what method was used to make this estimation. Researchers have provided uncertainty on PTR results in the past, but have not provided adequate explanation of how these values were obtained[13]. Since a thorough uncertainty analysis of PTR has not been conducted, there exists ample motivation for the present work. The uncertainty definition and calculations herein have been conducted according to the methods of Coleman and Steele[14], who in turn 


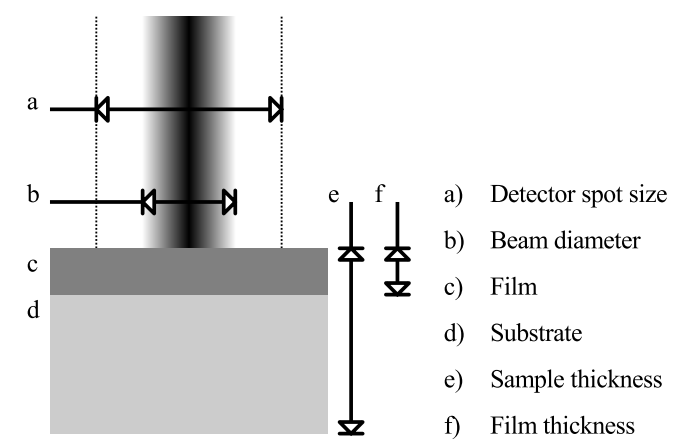

Figure 2: Diagram displaying two layer sample with laser spot and detection area's identified

follow the recommendations of the International Standards Organization (ISO).

The scope of this uncertainty analysis is limited to frequency-domain front-detection PTR on a opaque two-layer sample. Despite this narrow scope, the results of this analysis can provide insight to the uncertainty behavior of similar methods since the uncertainty propagation and curve fitting process is largely independent of how a frequency scan was measured. Such information can be of great value during experimental design, since it is often uncertainty which defines the practical envelope of an experiment. Quantifying experimental uncertainty requires specific knowledge of both uncertainty sources and the effects of data reduction on those uncertainties. Sensitivity studies can be used to identify the sources of uncertainty, and give some information about how that uncertainty propagates.

As in many experiments, PTR relies on measurements which are reduced to compute desired quantities which cannot be measured directly. Some method must be used to propagate the measurement uncertainties to the calculated value. The Monte Carlo method was used for uncertainty propagation in this study because a curve fit is used to compute the sample's thermal properties from a frequency scan.

Uncertainty analysis was conducted with input distributions assumed for layer thicknesses, properties, frequency, and phase measurement capabilities. This analysis was performed to understand the expected uncertainties in PTR measurements and to compare experimental considerations based thereon. Linear and logarithmic spacing of sampled frequencies was investigated to determine which better resolves the phasefrequency relationship. Additionally, a comparison of fits assuming 1D or 2-D thermal wave fields was conducted.

As stated, this study focuses on an opaque sample composed of two layers referred to as the film and substrate. Referring to the top layer as the film does not imply that it is thin, since this study was conducted without any thin-film assumptions made. Figure 2 diagrams the two layer sample studied, and also illustrates heating and detection areas on the sample. 


\section{Theoretical Models}

The concept of thermal wave fields is what makes photothermal methods possible, since periodic excitation is common to nearly every such method. Despite a solid foundation of well-known concepts such as heat conduction and harmonic analysis, thermal wave fields are not commonly studied as part of a traditional heat transfer curriculum. Thus, this section begins with a review of the governing equations of thermal wave propagation and concludes with a non-dimensionalization of these equations.

\subsection{Governing Equations}

The governing differential equation for modulated PTR is the thermal wave field equation as given in (1), where $T$ is the temperature of the sample, $k$ is the thermal conductivity, $Q(r, \omega)$ is defined as the Fourier transform of the volumetric thermal source, $\omega$ is the angular frequency, and $\sigma$ is called the thermal wave number and defined as in equation (2).

$$
\begin{aligned}
& \nabla^{2} T(\mathbf{r}, \omega)-\sigma^{2}(\omega) T(\mathbf{r}, \omega)=-\frac{1}{k} Q(\mathbf{r}, \omega) \\
& \sigma_{j}=\frac{1+i}{\mu_{j}}
\end{aligned}
$$

The thermal diffusion length $\mu$ is given by (3), where $\alpha$ is the thermal diffusivity; this length scale is defined by the point at which a thermal wave's amplitude will have diminished to $e^{-1}$ times its original intensity.

$$
\mu_{j}=\sqrt{\frac{2 \alpha_{j}}{\omega}},
$$

The thermal-wave-field equation is obtained by taking the Fourier transform of the heat conduction equation, then separating the periodic and non-periodic components of temperatures as provided in previous work by Mandelis[15]. The solution to (1) used in this study was obtained by use of Green's functions, and shown in (4) where $\Theta$ is the complex temperature. The magnitude of $\Theta$ corresponds to the amplitude and the argument represents the phase of the surface temperature. $Q$ is defined as the heating surface flux, $\rho$ is defined by (5) with $b$ and $R$ defined in (6) and (7) respectively.

$$
\begin{aligned}
\Theta & =\frac{Q}{4 k_{1} \sigma_{1}}\left[\frac{\left(1+R_{1}\right)\left(1+\rho_{21} e^{-2 \sigma_{1} L_{1}}\right)}{1-R_{1} \rho_{21} e^{-2 \sigma_{1} L_{1}}}\right] \\
\rho_{2,1} & =\frac{\left(1-b_{2,1}\right)+R_{2}\left(1+b_{1,2}\right) e^{-2 \sigma_{2} L_{2}}}{\left(1+b_{2,1}\right)+R_{2}\left(1-b_{1,2}\right) e^{-2 \sigma_{2} L_{2}}} \\
b_{i, j} & =\frac{\sqrt{k_{i} C_{i}}}{\sqrt{k_{j} C_{j}}} \\
R_{j} & =\frac{k_{j} \sigma_{j}-h_{j}}{k_{j} \sigma_{j}+h_{j}}
\end{aligned}
$$




\subsection{Non-dimensional Parameters}

A dimensional analysis was performed on the solution given in equation (4) to reduce the number of parameters and obtain a deeper understanding of the physics involved. The dimensionless parameters found are given in equations (8) through (11).

$$
\begin{aligned}
& F_{o_{j}}=\frac{k_{j}}{\omega C_{j} L_{j}^{2}}=\frac{\alpha_{j}}{\omega L_{j}^{2}}, \\
& B i_{j}=\frac{L_{j} h_{j}}{k_{j}}, \\
& \pi_{1}=\frac{C_{1} k_{1}}{C_{2} k_{2}} \\
& \pi_{2}=\frac{Q}{\Theta_{o} h_{1}}
\end{aligned}
$$

The first parameter given by equation (8) is a dimensionless time scale commonly found in heat transfer known as the Fourier number, and defined by the ratio of heat conduction rate to thermal energy storage[16]. Equation (9) is also a common dimensionless parameter known as the Biot number, which characterizes the ratio of convection heat transfer to conduction heat transfer[16]. Equation (10) defines the third dimensionless number being defined as $\pi_{1}$ which is the ratio of effusivities. This value was found by Almond and Patel to quantify the thermal mismatch between the two materials, and determines the magnitudes of the reflection and transmission of thermal waves[2]. The final parameter is defined in equation (11) as $\pi_{2}$. Expressing eq (4) in these parameters, the non-dimensionalized thermal wave field solution becomes equations (12) through (14).

$$
\begin{aligned}
\Theta^{*}= & \frac{\pi_{2} B i_{1} \sqrt{F O_{1}}}{2 \sqrt{2}(1+i)}\left(\frac{\left(1+R_{1}\right)\left(1+\rho_{21} e^{-\frac{2(i+1)}{\sqrt{2 F o_{1}}}}\right)}{1-R_{1} \rho_{21} e^{-\frac{2(i+1)}{\sqrt{2 F o_{1}}}}}\right) \\
\rho_{21}= & \frac{\left(1-\sqrt{\frac{1}{\pi_{1}}}\right)+R_{2}\left(1+\sqrt{\pi_{1}}\right) e^{-\frac{2(1+i)}{\sqrt{2 F o_{2}}}}}{\left(1+\sqrt{\frac{1}{\pi_{1}}}\right)+R_{2}\left(1-\sqrt{\pi_{1}}\right) e^{-\frac{2(1+i)}{\sqrt{2 F o_{2}}}}} \\
R_{j}= & \frac{(1+i)-B i_{j} \sqrt{2 F o_{j}}}{(1+i)+B i_{j} \sqrt{2 F o_{j}}}
\end{aligned}
$$

\section{Methods}

In this work the Monte Carlo uncertainty propagation method is used to examine the expected uncertainties of PTR measurement, as well as to compare variations in that can be made in the curve fitting process. These are the spacing of frequencies using during scans and the model used which is fit against the data. 


\subsection{Monte Carlo Uncertainty Estimation}

Any experiment which requires data to be combined to compute the final result requires a data reduction process (DRP), which can be either and equation (DRE) or algorithm (DRA). Two methods of propagating measurement uncertainties through a data reduction process are commonly used in practice, being the Taylor's series method and the Monte Carlo method. The first method finds primary use due to its simplicity, both theoretical and practical. The second method, that of Monte Carlo uncertainty propagation, has found favor more recently as personal computers have become sufficiently powerful to handle the computational costs associated with it $[17,18,19]$. As the name implies, this method is a stochastic estimation of the uncertainty through repeated application of the data reduction process.

In the Monte Carlo method, before an experiment is run, hypothetical measurement distributions are generated based on prior knowledge and their expected uncertainties. These values can be measured with existing equipment or based on specifications, but in either case form an input to the Monte Carlo process. For each set of input values, the result of the DRP is computed; the statistical properties of the output distribution are then used to describe the uncertainty of the result. Because the actual input values for each Monte Carlo sample are randomly selected from their parent distributions, their reduction is also random, and belongs to the parent distribution of the computed results. When enough samples are taken, the statistical characteristics of the input and output distributions will converge to stable values, which are the input and output uncertainties respectively. This process can be very computationally intensive, and for many non-trivial DRP's will require significant computer resources to make possible. The current study was conducted on a cluster computer with 1496 cores and took a full week to run.

As a post-processing step to an experiment, Monte Carlo can be applied to actually measured data sets instead of hypothetical distributions. In this case the output distribution will reflect the true propagated uncertainty from the experiment. Contrasting this to the more common Taylor's series method some important differences can be noted. Firstly, in both pre and post experimental uncertainty estimation the correlations of the variables are considered. In the pre-experimental case, correlated distributions can be used, or at least any correlations from the DRP will be present, whereas in the post-experimental case any correlations present in the data will be taken into account. This capability comes from processing all the data with the DRP instead of applying it merely to the means of the measured data.

The thermal properties measured by PTR are computed through fitting analytical models to the complex surface temperature as a function of frequency, normally called a frequency scan. Because these models are nonlinear, the curve parameters cannot be computed directly but are instead obtained from a minimization of the difference between the model and data. By using a minimization process to reduce the measured data the PTR data reduction process is actually an algorithm, which precludes the use 
of the Taylor's series method of uncertainty propagation. Sensitivity studies can be used to obtain the derivatives needed the the Taylor's series method, but by using the Monte Carlo method instead correlations can be included and the sensitivity studies are effectively done automatically.

\subsection{Experimental Design Considerations}

The thermal wave phenomena is often unfamiliar to researchers, and the result of changing a specific parameter is not always intuitive. These effects are also crucial to experimental uncertainty. Typically with the frequency-scan PTR method, thermal effusivity and diffusivity are measured. For these reasons a sensitivity analysis was conducted on these properties with respect to each of the input variables. To calculate these sensitivities, the physics were modeled with the correct value of the input parameter. Then the diffusivity and effusivity of the film were calculated with a specified variation of the input parameter. For the example of film thickness, equation (4) was used with the correct thickness of the film to obtain a response of $\Theta$. Then when equation (4) was fitted to the response of $\Theta$ for diffusivity and effusivity a different value was used for the film thickness. This difference between the input value use for the physics simulation and curve fit results in error from the fit. The results from this study will be provided later, and compared to those obtained from the dimensional analysis presented earlier.

Two matters of experimental design are considered in this work. The first is the spacing of the data points taken in the frequency scans: should they be linearly or logarithmically spaced? The second matter is which model to use for the curve fits in regimes which are known to be 2-D, the 1-D model or the 2-D. Both of these questions can be answered before data is ever taken by using Monte Carlo uncertainty analysis.

In experiments continuous frequency data is impossible, and attempting to obtain close to continuous data is impractical. This makes it necessary to determine specific frequencies to measure the phase. By looking at equation (3) it may be supposed that the frequencies should be spaced logarithmically to match the square root of the frequency in the thermal diffusion length, but inspection of the solution in (4) makes the matter less clear. To be certain which method to use, it must be tested by fitting scans done with the same number of frequencies but differing spacings and then comparing the resulting uncertainties. The results of this process are given later.

Laser-heated PTR systems will produce a 2-D axisymetric thermal response in the

sample; the solution to this heating by a Gaussian laser beam is given by (15) as derived by Mandelis[15].

$$
\begin{aligned}
\Theta(r, \omega)= & \frac{F_{o} W^{2}}{2 k_{1}} \int_{0}^{\infty} \frac{\left[1+R_{1}(\lambda)\right]\left[1+\rho_{21}^{(N)}(\lambda) e^{-s_{1}\left(2 L_{1}\right)}\right.}{s_{1}(\lambda)\left[1-R_{1}(\lambda) \rho_{21}^{(N)}(\lambda) e^{-2 s_{1} L_{1}}\right]} \\
& e^{-(\lambda W / 2)^{2}} J_{0}(\lambda r) \lambda d \lambda \\
s_{j}(\lambda)= & \sqrt{\lambda^{2}+\sigma^{2}}
\end{aligned}
$$




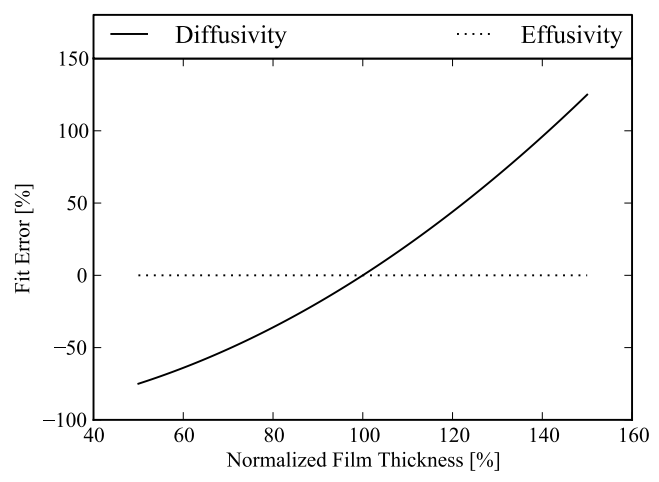

Figure 3: Sensitivity plot of diffusivity and effusivity as the "measured" value for layer thickness is varied in the curve fitting process.

This analytic solution is valuable, but is computationally much more expensive to evaluate in the fitting process than equation (4), being due to the numerical integration. This is the motivation for researchers to make a 1-D heat transfer assumption when solving for thermal properties. There are two scenarios where this 1-D heat transfer assumption is accurate. The first consists of the detection area being much smaller than the heating spot size. In this case, the detector observes only uniform heating of a sample and it is indeed 1-D. The second case is where the detector observes a much larger area than the heating spot size. The mathematical proof for this has been provided in the literature[20]. It is not always feasible to attain one of these scenarios; the range of interest is where the detection size is comparable to the size of the heating laser spot. Results of the error associated with using the 1-D fit in this range are provided below.

\section{Results}

\subsection{Sensitivity Analysis}

The results of the sensitivity analysis are displayed in figures 1-3. The most unique result is the nonlinear relationship between uncertainties of film diffusivity and film thickness shown in figure 3. This non-linear relationship can be explained by equation (8) in the dimensional analysis where $\omega$ and $L$ are related non-linearly. From a closer inspection of these dimensionless parameters it is predicted that all other relationships should be linear if correlated at all. This prediction was confirmed from the sensitivity study conducted, and the results are presented in table 1.

Because of the non-linear relationship between the uncertainty of the film thickness and that of the computed diffusivity, it is expected that the distribution of the diffusivity will be skewed. As can be seen in figure 4 this is the case, with the diffusivity noticeably deviating from a normal distribution. Although the difference is slight, it is definite, and would be greater if the non-linearity were more extreme. 


\begin{tabular}{|c|c|c|}
\hline Parameter & $\alpha$ Sensitivity & $\epsilon$ Sensitivity \\
\hline Film Thickness & Non-Linear & Uncorrelated \\
\hline Substrate $\alpha$ & Uncorrelated & Uncorrelated \\
\hline Substrate $\epsilon$ & Uncorrelated & Linear $(1: 1)$ \\
\hline Frequency & Linear $(1: 1)$ & Uncorrelated \\
\hline
\end{tabular}

Table 1: Correlations between non-dimensional parameters

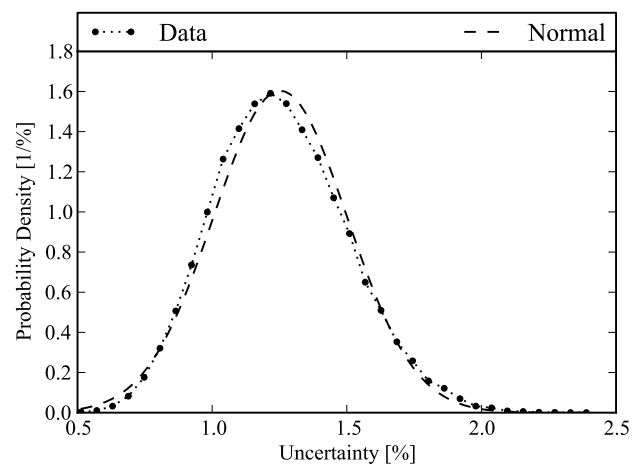

Figure 4: Histogram of actual output uncertainty distribution due to film thickness uncertainty plotted against a normal distibution with the same mean and standard deviation.

\subsection{Overall Uncertainty}

The overall uncertainty from a PTR measurement is estimated using the Monte Carlo method byt selecting parameters that best-describe the experimental setup to be used. Since this work is largely hypothetical a group of six different materials was selected to represent the spectrum of thermal properties commonly found in nature. These six materials were then used as either the film material or the substrate material in simulations, resulting in 30 combinations since a material used for both film and substrate could not be measured using PTR. The materials selected for use are the following: Zr, Ge, Si, Al, Au, and Ag.

The physical parameters and input uncertainties used for this study are listed in table 2, and were chosen to closely match the PTR system here at USU, some of the data even having been directly measured using it. The overall uncertainty results are found in table 3, and are split into bias and precision for the diffusivity and effusivity.

The thermal conductivity $(k)$ and volumetric specific heat $(C)$ can be computed from the thermal diffusivity $(\alpha)$ and thermal effusivity $(\epsilon)$ be the following relations: $k=\epsilon \cdot \sqrt{\alpha}$, and $C=\epsilon / \sqrt{\alpha}$. Using Taylor's series uncertainty propagation, the uncertainties can be computed as $u=\sqrt{u_{\epsilon}^{2}+u_{\alpha}^{2} / 4}$ in both cases.

Because of the difficulty in using 30 results on a plot simultaneously, all the 
Table 2: Characteristic PTR setup parameters

\begin{tabular}{l|c|c} 
Parameter & Value & Units \\
\hline Sample Thickness & 2000.0 & $\mu$ \\
Film Thickness & 10.0 & $\mu$ \\
Beam Diameter & 1000.0 & $\mu$ \\
Detector Spot Diameter & 200.0 & $\mu$ \\
Frequency Uncertainty & 0.0083 & $\mathrm{~Hz}$ \\
Film Thickness Uncertainty & 5.0 & $\%$ \\
Phase Uncertainty (Lock-in) & 1.0 & $\circ$ \\
Phase Uncertatinty (Transfer Function) & 0.5 & $\circ$ \\
Diffusivity Unceratinty & 4.75 & $\%$ \\
Effusivity Unceratinty & 3.0 & $\%$
\end{tabular}

Table 3: Full dataset for overall uncertainty

(a) Diffusivity bias uncertainty in percent $\quad$ (b) Diffusivity precision uncertainty in percent

\begin{tabular}{|c|c|c|c|c|c|c|c|c|c|c|c|c|c|}
\hline & $\mathrm{Zr}$ & Ge & $\mathrm{Si}$ & $\mathrm{Al}$ & $\mathrm{Au}$ & $\mathrm{Ag}$ & & $\mathrm{Zr}$ & Ge & $\mathrm{Si}$ & $\mathrm{Al}$ & $\mathrm{Au}$ & $\mathrm{Ag}$ \\
\hline $\mathrm{Zr}$ & & 0.39 & 0.33 & 0.32 & 0.31 & 0.31 & $\mathrm{Zr}$ & & 10.3 & 10.2 & 10.2 & 10.2 & 10.2 \\
\hline $\mathrm{Ge}$ & 0.16 & & & & 0 & & $\mathrm{Ge}$ & 10.2 & & & & & 10.2 \\
\hline $\mathrm{Si}$ & ( & 0.15 & & 0.41 & 0.3 & 0.3 & & 10.2 & 10.2 & & 10.3 & & 10.2 \\
\hline $\mathrm{Al}$ & & 0.21 & 0.15 & & 0.78 & 0.48 & $\mathrm{Al}$ & 10.2 & 10.2 & 10.2 & & 10.4 & 10.3 \\
\hline $\mathrm{Au}$ & & 0.22 & 0.18 & 0.03 & & 0.81 & $\mathrm{Au}$ & 10.2 & 10.2 & 10.2 & 10.3 & & 10.5 \\
\hline$\Delta \alpha$ & 23 & 0.22 & 0.19 & 0.11 & 0.01 & & $\mathrm{Ag}$ & 10.2 & 10.2 & 10.2 & 10.2 & 10.4 & \\
\hline
\end{tabular}

(c) Effusivity bias uncertainty in percent

(d) Effusivity precision uncertainty in percent

\begin{tabular}{|c|c|c|c|c|c|c|c|c|c|c|c|c|c|}
\hline & $\mathrm{r}$ & $\mathrm{Ge}$ & $\mathrm{Si}$ & $\mathrm{Al}$ & $\mathrm{Au}$ & $\mathrm{Ag}$ & & $\mathrm{Zr}$ & $\mathrm{Ge}$ & $\mathrm{Si}$ & $\mathrm{Al}$ & $\mathrm{Au}$ & $\mathrm{Ag}$ \\
\hline $\mathrm{Zr}$ & & 0.10 & 0.13 & 017 & 0.17 & & $\mathrm{Zr}$ & & & & & 2.18 & \\
\hline $\mathrm{Ge}$ & 0.12 & & 0.09 & 0.14 & 0.16 & 0.15 & $\mathrm{Ge}$ & 1.19 & & 1.16 & & & 74 \\
\hline $\mathrm{Si}$ & 0.11 & 0.13 & & 0.1 & & & $\mathrm{Si}$ & & 1.1 & & & & .35 \\
\hline $\mathrm{Al}$ & 0 & 0.14 & 0.14 & & 0.15 & 0.1 & $\mathrm{Al}$ & 2.03 & 1.4 & 1.1 & & 1.09 & 1.11 \\
\hline $\mathrm{Au}$ & 2 & 0.15 & 0.14 & 0.11 & & 0.14 & $\mathrm{Au}$ & 2.35 & 1.6 & 1.24 & & & 1.08 \\
\hline & .29 & 0.18 & 0.15 & 0.12 & 0.11 & & $\mathrm{Ag}$ & 2.69 & 1.84 & 1.36 & 1.14 & 1.12 & \\
\hline
\end{tabular}

Table 4: Uncertainties on computed conductivity or specific heat in percent

\begin{tabular}{c|cccccc} 
& $\mathrm{Zr}$ & $\mathrm{Ge}$ & $\mathrm{Si}$ & $\mathrm{Al}$ & $\mathrm{Au}$ & $\mathrm{Ag}$ \\
\hline $\mathrm{Zr}$ & & 5.26 & 5.31 & 5.46 & 5.56 & 5.69 \\
$\mathrm{Ge}$ & 5.24 & & 5.26 & 5.30 & 5.35 & 5.40 \\
$\mathrm{Si}$ & 5.31 & 5.24 & & 5.26 & 5.27 & 5.29 \\
$\mathrm{Al}$ & 5.49 & 5.31 & 5.24 & & 5.35 & 5.26 \\
$\mathrm{Au}$ & 5.62 & 5.36 & 5.25 & 5.29 & & 5.37 \\
$\mathrm{Ag}$ & 5.77 & 5.43 & 5.28 & 5.25 & 5.31 &
\end{tabular}




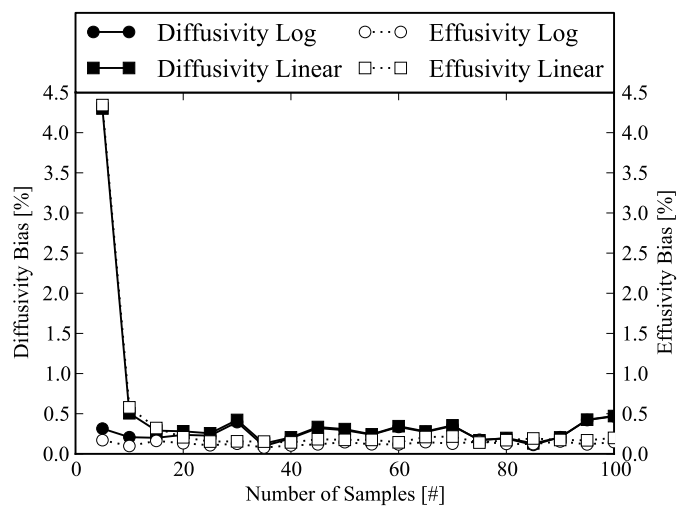

Figure 5: Comparison of logarithmic and linear frequency spacing. Bias uncertainty in effusivity and diffusivity are plotted as a function of the number of frequencies sampled.

subsequent results in this work are reported with a single value that is the mean of the 30 individually calculated values. Since the results are largely comparative of one method to another, this simplification of representation is deemed acceptable. When examined individually, the results for each combination of materials follow the trend of the mean values.

\subsection{Logarithmic vs. Linear Data Spacing}

Two methods that have been used by researchers are linear and log spacing of sampled frequencies. If large numbers of data are taken with either spacing technique the fitting process will obtain the same result. This comparison was conducted to determine which spacing method will obtain more accurate results from the same number of data points. It can be seen from figures 5 and 6 that as the number of samples taken increases the results for linear and logarithmic spacing of the frequencies converge to the same result as expected. However, at low number of samples logarithmic spacing of samples obtains significantly better results.

\subsection{1-D vs 2-D Fitting Procedure}

The results from fitting equation (4) in a regime where the exact solution is given by equation (15) are provided in figures 7 and 8 . The process was conducted with a range of beam diameters while keeping the detection area of 100 micron diameter concentric with the laser beam. As discussed earlier, it is important to note that the equations (4) and (15) yield the same results in the range where the detection area is much smaller than the beam diameter, and also when the detection area becomes significantly larger than the beam diameter.

It is shown in figure 7 that fitting equation (4) or (15) do indeed obtain the same results at the limiting cases where the detection area is much larger or much smaller than 


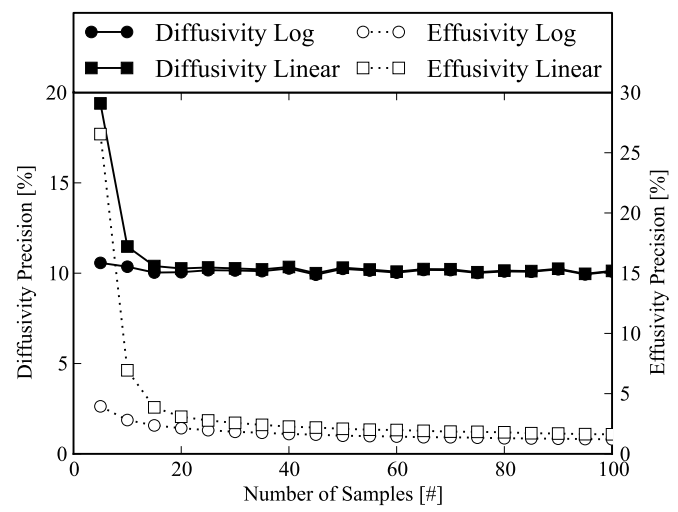

Figure 6: Comparison of logarithmic and linear frequency spacing. Precision uncertainty in effusivity and diffusivity are plotted as a function of the number of frequencies sampled.

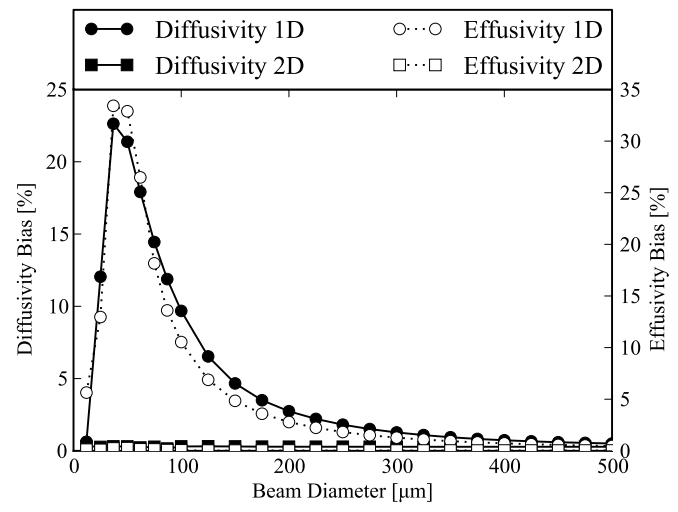

Figure 7: Effusivity and diffusivity bias uncertainty from 1-D and 2-D fits vs beam diameter

the heating spot size. It is also important to note the maximum bias uncertainty occurs from fitting equation (4) when the beam diameter is approximately half the diameter of the detection area.

\section{Conclusions}

The results from this work provide several important conclusions relating to uncertainty associated with PTR, and also provides guidelines on how to minimize uncertainty while testing.

The sensitivity results are among the most valuable in this study. These results provide direct information on how the variables affect each other. Therefore, it also provides information on how the uncertainty of the variables relates to the results[14]. One can imagine a case where an experimentalist was interested in measuring the 


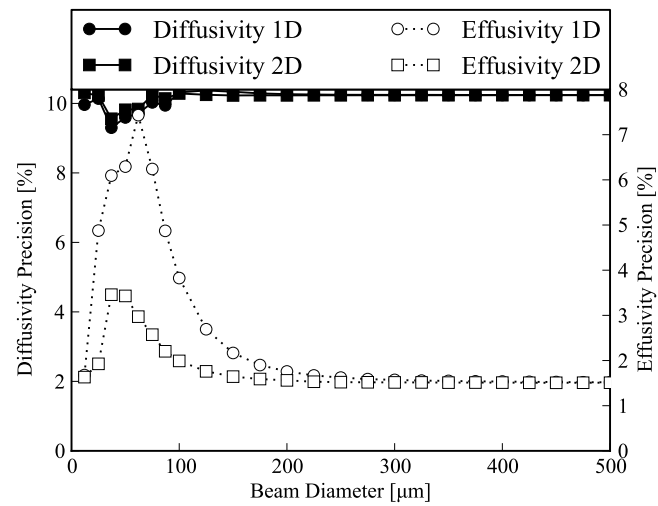

Figure 8: Effusivity and diffusivity precision uncertainty from 1-D and 2-D fits vs beam diameter

film diffusivity, but did not have an accurate value for substrate effusivity. Because of these sensitivity results the experimentalist knows that the substrate effusivity and film diffusivity are not correlated, and therefore does not need to be concerned about the accuracy of the effusivity value for those specific results.

From the logarithmic vs linear frequency spacing tests performed, it can be concluded that logarithmic spacing of frequency data points should be performed. Given the same number of data points over a set frequency range the logarithmic spacing always obtained better results.

\section{References}

[1] Svein Otto Kanstad Erik Nordal. Photothermal radiometry. Physica Scripta, 20:659-662, 1979. 1

[2] P. M Patel D. P. Almond. Photothermal Science and Techniques. Chapman \& Hall, 1996. 1, 2.2

[3] K. Horne, H. Ban, A. Mandelis, and A. Matvienko. Photothermal radiometry measurement of thermophysical property change of an ion-irradiated sample. Materials Science and Engineering: $B, 2011.1$

[4] R. Santos and LCM Miranda. Theory of the photothermal radiometry with solids. Journal of Applied Physics, 52(6):4194-4198, 1981. 1

[5] A. Mandelis and Z. Chen. Lock-in rate-window thermomodulation (thermal wave) and photomodulation spectrometry. Review of scientific instruments, 63(5):2977-2988, 1992. 1

[6] E. MacCormack, A. Mandelis, M. Munidasa, B. Farahbakhsh, and H. Sang. Measurements of the thermal diffusivity of aluminum using frequency-scanned, transient, and rate window photothermal radiometry. theory and experiment. International journal of thermophysics, 18(1):221-250, 1997. 1

[7] A. Salazar, E. Apinaniz, A. Mendioroz, R. Celorrio, et al. Simultaneous measurement of thermal diffusivity and optical absorption coefficient using photothermal radiometry. ii multilayered solids. Journal of Applied Physics, 110(3):033516-033516, 2011. 1

[8] A. Rosencwaig, J. Opsal, W.L. Smith, and DL Willenborg. Detection of thermal waves through optical reflectance. Applied Physics Letters, 46(11):1013-1015, 1985. 1

[9] JC Murphy and LC Aamodt. Photothermal spectroscopy using optical beam probing: mirage effect. Journal of Applied Physics, 51(9):4580-4588, 1980. 1 
[10] A. Rosencwaig and A. Gersho. Theory of the photoacoustic effect with solids. Journal of Applied Physics, 47(1):64-69, 1976. 1

[11] MA Olmstead, NM Amer, S. Kohn, D. Fournier, and AC Boccara. Photothermal displacement spectroscopy: an optical probe for solids and surfaces. Applied Physics A: Materials Science 83 Processing, 32(3):141-154, 1983. 1

[12] A. Mandelis and M.M. Zver. Theory of photopyroelectric spectroscopy of solids. Journal of applied physics, 57(9):4421-4430, 1985. 1

[13] J. Bodzenta, A. Kaźmierczak-Bałata, and J. Mazur. Photothermal methods for determination of thermal properties of bulk materials and thin films. Central European Journal of Physics, $8(2): 207-220,2010.1$

[14] H.W. Coleman and W.G. Steele. Experimentation, validation, and uncertainty analysis for engineers. Wiley, 2009. 1, 5

[15] Andreas Mandelis. Diffusion-Wave Fields Mathematical Methods and Green Functions. SpringerVerlag, 2001. 2.1, 3.2

[16] Incropera, DeWitt, Bergman, and Lavine. Fundamentals of heat and mass transfer. Wiley, 2007. 2.2

[17] K. Horne, H. Ban, R. Fielding, and R. Kennedy. Monte carlo uncertainty estimation for an oscillating-vessel viscosity measurement. Metrologia, 49(4):577, 2012. 3.1

[18] S. Greenland. Sensitivity analysis, monte carlo risk analysis, and bayesian uncertainty assessment. Risk Analysis, 21(4):579-584, 2002. 3.1

[19] M.G. Cox and B.R.L. Siebert. The use of a monte carlo method for evaluating uncertainty and expanded uncertainty. Metrologia, 43(4):S178, 2006. 3.1

[20] E. Marín. Basic principles of thermal wave physics and related techniques. 2009. 3.2 